\title{
Fine Structure of Open Shell Diatomic Molecules in Combined Electric and Magnetic Fields
}

\author{
Martin Gärttner, ${ }^{1, *}$ Juan J. Omiste, ${ }^{1}$ Peter Schmelcher, ${ }^{2}$ and Rosario González-Férez ${ }^{1, \dagger}$ \\ ${ }^{1}$ Instituto Carlos I de Física Teórica y Computacional, and Departamento de Física Atómica, \\ Molecular y Nuclear, Universidad de Granada, 18071 Granada, Spain \\ ${ }^{2}$ Zentrum für Optische Quantentechnologien, Universität Hamburg, \\ Luruper Chaussee 149, Hamburg, 22761, Germany
}

(Dated: July 17, 2018)

\begin{abstract}
We present a theoretical study of the impact of an electric field combined with a magnetic field on the rotational dynamics of open shell diatomic molecules. Within the rigid rotor approximation, we solve the time-independent Schrödinger equation including the fine-structure interactions and the $\Lambda$-doubling effects. We consider three sets of molecule specific parameters and several field regimes and investigate the interplay between the different interactions identifying the dominant one. The possibility of inducing couplings between the spin and rotational degrees of freedom is demonstrated. ine structure, rotational motion, magnetic field, electric field
\end{abstract}

\section{INTRODUCTION}

The control and manipulation of all molecular degrees of freedom, i.e., the center of mass, electronic, rotational and vibrational motion, is an ambitious goal in modern molecular physics. Ensembles of cold and ultracold molecules present a paradigm in this context. Within the last decade, many experimental techniques, based on the use of external fields, have been developed to create samples of cold and ultracold molecules [1 -5$]$. Currently, different species of cold molecules are becoming available [6 -9] with a special focus on heteronuclear alkali dimers [4, 10 13]. These experimental efforts should be accompanied by theoretical studies to understand how external fields modify the internal structure of these systems.

In the recent years, a series of studies of the rotational spectrum of several diatomic molecules in a ${ }^{1} \Sigma$ electronic state exposed to combined electric and magnetic fields were performed [14, 15]. These systems are characterized by two distinct energy scales associated to the rotational degrees of freedom and the hyperfine structure. The next key structural ingredient is given by the electronic spin and orbital angular momentum, i. e., molecules in a ${ }^{2} \Sigma$ or ${ }^{2} \Pi$ electronic state. These systems are ideal to analyze the interplay between the spin-orbit coupling and the rotational structure in the presence of external fields. Furthermore, the Zeeman and Stark effects might be comparable for moderate field strengths. At the end of the 1990 s, polar ${ }^{2} \Sigma$ molecules were investigated in congruent fields with the focus on their directional properties and the possibility of trapping them [16]. Recently, open shell diatomic molecules, in ${ }^{2} \Pi$ electronic states, exposed to combined electric and magnetic fields have been also analyzed from both theoretical and experimental point of

\footnotetext{
* Max Planck Institut für Kernphysik, Saupfercheckweg 1, 69117 Heidelberg, Germany

$\dagger$ rogonzal@ugr.es
}

views 17, 18]. The avoided crossings in the field-dressed spectrum of $\mathrm{OH}$ were used to transfer population between two states of opposite parity, and the trap dynamics was observed in combined fields [18]. The theoretical analysis has been carried out under the assumption that the total angular momentum remains approximately constant, and, therefore, only the coupling between different $\Lambda$ doublet states has been taken into account [17, 18].

The present study goes beyond this approximation and aims at an extended approach to the rotational motion of an open shell dimer in a ${ }^{2} \Pi$ electronic state in combined electric and magnetic fields. In particular, we describe it within the rigid rotor approximation including the fine structure interactions and the $\Lambda$-doubling effects. Taking as prototype examples the $\mathrm{LiO}$ and $\mathrm{OH}$ radicals in their ${ }^{2} \Pi$ electronic ground state, we explore a wide range of field strengths and two different regimes characterized by: i) the field-dressed dynamics taking place within a certain rotational manifold; and ii) the possibility of mixing states in neighbouring rotational manifolds of the ${ }^{2} \Pi_{3 / 2}$ fine structure component. Our focus is on the energy shifts, the directional properties, and the hybridization of the angular motion as either the electric or magnetic field strengths, or the inclination angle between them is varied. We also investigate field-induced couplings between levels of the fine structure components ${ }^{2} \Pi_{1 / 2}$ and ${ }^{2} \Pi_{3 / 2}$ by using the external fields. For a molecule with small rotational splitting, we show that the coupling between the spin and rotational degrees of freedom could be achieved at realistic field strengths. It has been shown recently, that the spin-rotational coupling can be exploited for tailoring the interactions between ultracold molecules [19 21]. We show that similarly the presence of quasi-degenerate $\Lambda$-doublets of states provide versatile tools for shaping the properties of ultracold molecules.

The paper is organized as follows. In section II we define our working Hamiltonian. In section III we discuss the numerical results for two diatomic molecules with a ${ }^{2} \Pi$ electronic ground state, $\mathrm{OH}$ and $\mathrm{LiO}$, as the field parameters are modified. In particular, we explore three 

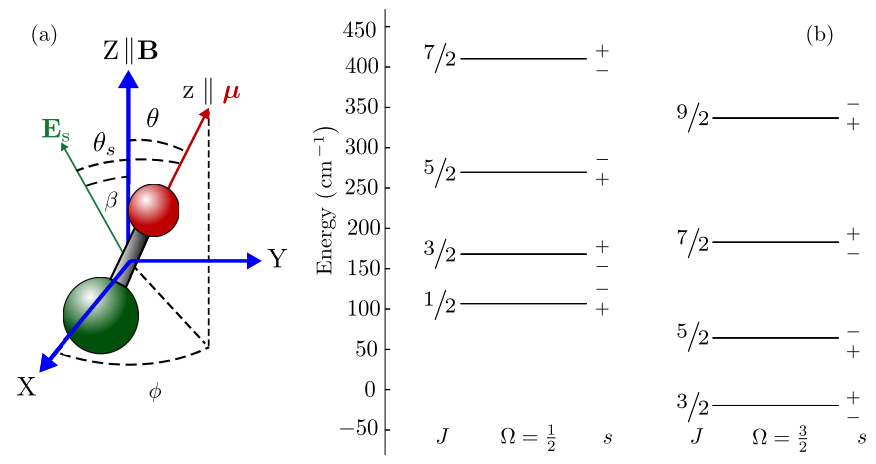

FIG. 1. (a) Laboratory fixed frame, Euler angles, schematic field configuration and diatomic molecule. (b) Field-free level structure of the $\mathrm{OH}$ molecule. The energy splitting due to the $\Lambda$-doubling is not visible on the scale of this figure, the plus and minus signs indicate if the even or odd component of a doublet is energetically higher or lower.

different cases: (i) for fixed magnetic field and four inclination angles, we vary the electric field strength; (ii) for fixed electric field and two inclination angles, the magnetic field is increased; (iii) for fixed magnetic and electric field strengths, the angle between them is continuously changed from $0^{\circ}$ to $90^{\circ}$. Finally, for a model system, we investigate the possibility of mixing states from two different fine structure components. The conclusions are provided in section IV.

\section{THE HAMILTONIAN OF A LINEAR RIGID ROTOR IN EXTERNAL FIELDS}

We consider a polar linear molecule in a ${ }^{2} \Pi$ electronic state exposed to a homogeneous static electric field and a homogeneous static magnetic field. The field configuration is illustrated in Figure 1: The magnetic field vector B points along the $Z$-axis of the laboratory fixed frame $(\mathrm{LFF})(X, Y, Z)$, and the dc field $\mathbf{E}_{\mathrm{s}}$ is contained in the $X Z$-plane forming an angle $\beta$ with the $Z$-axis. The $z$-axis of the molecule fixed frame (MFF) $(x, y, z)$ is defined by the permanent dipole moment of the molecule $\boldsymbol{\mu}$. These two frames are related by the Euler angles $\theta$ and $\phi$, cf. Figure 1 (a). The system is described within the rigid rotor approximation, assuming that the vibrational and electronic dynamics are not affected by the fields. We have included the fine structure interactions and the $\Lambda$ doubling effects, this last term being the largest second order correction for a $\Pi$-state [22]. The effects due to higher order relativistic and hyperfine interactions have been neglected. Thus, the effective Hamiltonian [22] reads

$$
H=H_{0}+H_{\mathrm{s}}+H_{\mathrm{z}}
$$

where $H_{0}$ is the field-free Hamiltonian, and $H_{\mathrm{s}}$ and $H_{\mathrm{z}}$ stand for the interaction with the static electric and magnetic fields, respectively. The field-free Hamiltonian is given by

$$
H_{0}=H_{\mathrm{rot}}+H_{\mathrm{so}}+H_{\mathrm{sr}}+H_{\Lambda d}
$$

where $H_{\text {rot }}$ is the rotational Hamiltonian [22]

$$
H_{\text {rot }}=\mathcal{B} \mathbf{N}^{2}=\mathcal{B}(\mathbf{J}-\mathbf{S})^{2}
$$

with $\mathbf{N}$ being the total angular momentum operator excluding spin, $\mathbf{J}$ the total angular momentum operator excluding nuclear spin, $\mathbf{S}$ the electronic spin, and $\mathcal{B}$ the rotational constant. The spin-orbit coupling term reads

$$
H_{\text {so }}=A T_{0}^{1}(\mathbf{L}) T_{0}^{1}(\mathbf{S})
$$

where $\mathbf{L}$ is the electronic orbital angular momentum and $A$ the spin-orbit constant. Note that we are using the spherical tensor notation [23]. The contribution due to the electronic spin-rotation coupling is given by

$$
H_{\mathrm{sr}}=\gamma T^{1}(\mathbf{N}) \cdot T^{1}(\mathbf{S})
$$

where $\gamma$ is the spin-rotation constant. For a ${ }^{2} \Pi$ electronic ground state, the $\Lambda$-doubling term reads

$$
H_{\Lambda d}=\sum_{m= \pm 1} e^{-2 i m \phi}\left(p T_{2 m}^{2}(\mathbf{S}, \mathbf{N})-q T_{2 m}^{2}(\mathbf{N}, \mathbf{N})\right)
$$

where $p$ and $q$ are the $\Lambda$-doubling parameters. The term $H_{\Lambda d}$ is a second order term causing a splitting between levels with different electronic angular momentum projection quantum numbers $\Lambda$. It is due to the mixing of rotational states with even and odd parity, and the corresponding ones in the $\Sigma$ electronic state 22].

The electric dipole moment couples to the static electric field, resulting in

$$
H_{\mathrm{s}}=-\boldsymbol{\mu} \cdot \mathbf{E}_{\mathrm{s}}=-\mu \mathrm{E}_{\mathrm{s}} \cos \theta_{\mathrm{s}}
$$

with $\mathbf{E}_{\mathrm{s}}=\mathrm{E}_{\mathrm{s}}(\sin \beta \hat{X}+\cos \beta \hat{Z})$, and $\mathrm{E}_{\mathrm{s}}$ being the electric field strength. The angle between the dipole moment $\boldsymbol{\mu}$ and this field is $\theta_{\mathrm{s}}$, cf. Figure 1, and $\cos \theta_{\mathrm{s}}=\cos \beta \cos \theta+$ $\sin \beta \sin \theta \cos \phi$ with $0^{\circ} \leq \beta \leq 180^{\circ}$.

The interaction with the magnetic field is given by

$$
H_{\mathrm{z}}=\mu_{B} \mathbf{B} \cdot\left(g_{L} \mathbf{L}+g_{s} \mathbf{S}\right)
$$

where $\mu_{B}=e \hbar / 2 m$ is the Bohr magneton, and $g_{L}$ and $g_{s}$, are the electron orbital and spin gyromagnetic ratios, respectively, which can be approximated by $g_{L} \approx 1$ and $g_{s} \approx 2$.

Here, we consider molecules having a spin-orbit constant $A$ larger than the rotational constant $\mathcal{B}$. For the description of these systems, the Hund's case (a) coupling is suited best [22]. The basis set is formed by eigenstates of the commuting operators $L_{z}, \mathbf{S}^{2}, S_{z}, \mathbf{J}^{2}, J_{Z}$, and $J_{z}$. The operators $L_{z}, J_{z}$ and $S_{z}$ are the projections of the electronic orbital angular momentum $\mathbf{L}$, total angular momentum $\mathbf{J}$ and spin $\mathbf{S}$ on the $z$-axis of the MFF, respectively, whereas $J_{Z}$ is the projection of $\mathbf{J}$ on the 
LFF $Z$-axis. Since this study is restricted to the vibrational ground state of the electronic ground state, the electronic and vibrational Hamiltonians have not been included. The eigenstates of this basis $\left|\Lambda S \Sigma J M_{J} \Omega\right\rangle$ are labeled by $\Lambda, S, \Sigma, J, M_{J}, \Omega$, with $\Omega=\Lambda+\Sigma$. For a ${ }^{2} \Pi$ electronic state, $S=1 / 2$ and $\Lambda= \pm 1$. Performing a transformation to a basis of parity eigenstates

$$
\begin{aligned}
\left|J M_{J} \Omega s\right\rangle & =\frac{1}{\sqrt{2}}\left(\left|\Lambda S \Sigma J M_{J} \Omega\right\rangle\right. \\
& \left.+s(-1)^{J-S}\left|-\Lambda S-\Sigma J M_{J}-\Omega\right\rangle\right)
\end{aligned}
$$

the label $\Lambda$ becomes obsolete and is replaced by the parity $s= \pm 1$. Thus, only $\Omega, J, M_{J}$, and $s$ are needed to uniquely label the states.

The field-free Hamiltonian is invariant under any rotation, and $J, M_{J}$ and the parity $s$, are good quantum numbers, whereas, $\Omega$ is not well defined. The field-free states having different $\Omega$ are coupled, and their labeling is based on the adiabatic limit of vanishing spin-rotational coupling. There are two manifolds of fine structure levels ${ }^{2} \Pi_{3 / 2}$ and ${ }^{2} \Pi_{1 / 2}$, each one consisting of several rotational levels (cf. Fig. 1 (b)). For molecules having a negative fine structure constant, $A<0$, e.g., the $\mathrm{OH}, \mathrm{LiO}$ and $\mathrm{NaO}$ molecules, the ground state has $\Omega=3 / 2$. The $\Lambda$ doubling splitting between states of different parity is small compared to the rotational splitting. The field-free states are degenerate in $M_{J}$.

The symmetries of this system are significantly reduced when the fields are applied. In the presence of a static electric field, the Hamiltonian is invariant under arbitrary rotations around the field axis $\mathcal{C}_{\mathbf{E}_{\mathbf{s}}}(\delta)$ and reflections in any plane containing the field axis, being $M_{J}$ a good quantum number if $\beta=0^{\circ}$. If only the magnetic field is applied, the symmetries of the Hamiltonian consist on arbitrary rotations around the $\mathrm{LFF} Z$-axis $\mathcal{C}_{Z}(\delta)$ and inversions with respect to the origin of coordinates. In this case, $M_{J}$ and $s$ remain as good quantum numbers. For parallel or antiparallel fields, the Hamiltonian is invariant under any arbitrary rotations around the field axis $\mathcal{C}_{Z}(\delta)$ and $M_{J}$ is a good quantum number. If the fields are perpendicular the reflection in the plane perpendicular to the magnetic field, $\sigma_{X Y}$ is a symmetry. For any other angle, i. e., $\beta \neq 0^{\circ}, 90^{\circ}$ or $180^{\circ}$, all the symmetries of the Hamiltonian are broken.

The time-independent Schrödinger equation associated to the Hamiltonian (1) is solved by a basis set expansion in terms of the functions (9). For reasons of addressability, we will label the field-dressed states as $\left|J M_{J} \Omega s\right\rangle$, even if $J, M_{J}$ or $s$ are not good quantum numbers. Thus, $\left|J M_{J} \Omega s\right\rangle$ refers to the level that is adiabatically connected as $\mathrm{E}_{\mathrm{s}}, \mathrm{B}$, and/or $\beta$ are modified with the field-free state $\left|J M_{J} \Omega s\right\rangle$.

\section{RESULTS}

The molecules $\mathrm{LiO}, \mathrm{NaO}, \mathrm{OH}$ and $\mathrm{NO}$ have ${ }^{2} \Pi$ electronic ground states. The parameters of the effective Hamiltonian are listed in Table I] 22, 24, 25]. For LiO 24] and $\mathrm{NaO}$ 25], the values of the spin-rotation constants are not available in the literature to the best of our knowledge, so we have estimated them using an approximation based on a pure precession hypothesis $\gamma \approx-p / 2$ [26 29]. In this work, we consider two of these molecules, $\mathrm{LiO}$ and $\mathrm{OH}$, as benchmarks to illustrate our results. While both molecules have spin-orbit constants of the same order of magnitude, the rotational constant of $\mathrm{OH}$ is around 15.4 times larger than in $\mathrm{LiO}$. For $\mathrm{OH}$, there are two rotational manifolds of ${ }^{2} \Pi_{3 / 2}$ energetically below the first rotational manifold with $J=1 / 2$ of ${ }^{2} \Pi_{1 / 2}$, see the states with $J=3 / 2$ and $5 / 2$ of ${ }^{2} \Pi_{3 / 2}$ in Figure 1 (b). Opposite to this, for LiO, 8 rotational manifolds with $\Omega=3 / 2$ have energies smaller than the rotational ground state of ${ }^{2} \Pi_{1 / 2}$. For both systems, the energy gap to the neighbouring $\Sigma$ electronic state is large enough, so that its influence can be described by the $\Lambda$-doubling [22]. The contribution of the hyperfine structure is negligible, being the contribution of the quadrupole moment for the $\mathrm{LiO}$ in the order of tenths of $\mathrm{MHz}$ 22, 30, 31]. Compared to $\mathrm{OH}, \mathrm{LiO}$ has a larger dipole moment and a smaller rotational constant, thus, the same electric field strength would provoke a larger impact on the $\mathrm{LiO}$ rotational dynamics.

TABLE I. Parameters of the effective Hamiltonian for the ${ }^{2} \Pi$ ground state of the $\mathrm{OH}, \mathrm{LiO}, \mathrm{NaO}$ and $\mathrm{NO}$ molecules.

\begin{tabular}{ccccc}
\hline \hline Molecule & $\mathrm{OH}$ & $\mathrm{LiO}^{\mathrm{a}}$ & $\mathrm{NaO}^{\mathrm{a}}$ & $\mathrm{NO}$ \\
\hline $\mathcal{B}\left(\mathrm{cm}^{-1}\right)$ & 18.535 & 1.204 & 0.422 & 1.696 \\
$A\left(\mathrm{~cm}^{-1}\right)$ & -139.051 & -111.672 & -107.151 & 123.15 \\
$\gamma\left(\mathrm{cm}^{-1}\right)$ & -0.119 & -0.105 & $-4.42 \times 10^{-2}$ & $-6.47 \times 10^{-3}$ \\
$p\left(\mathrm{~cm}^{-1}\right)$ & 0.235 & 0.210 & $8.84 \times 10^{-2}$ & $1.17 \times 10^{-2}$ \\
$q\left(\mathrm{~cm}^{-1}\right)$ & -0.039 & $-1.89 \times 10^{-3}$ & $6.23 \times 10^{-4}$ & $9.41 \times 10^{-5}$ \\
$\mu(\mathrm{D})$ & 1.655 & 6.5 & 8.7 & 0.159 \\
\hline \hline
\end{tabular}

a The spin-rotation constants of $\mathrm{LiO}[24]$ and $\mathrm{NaO}$ [25] molecules has been estimated using the approximation $\gamma \approx-p / 2[26[28]$.

\section{A. Influence of the electric-field strength}

We start by analyzing the impact of a static electric field taking $\beta=0^{\circ}$. We restrict this study to the lowest lying eight states with $\Omega=3 / 2$ and $J=3 / 2$. Note that they well represent the main physical features observed in the overall molecular dynamics, and similar behavior and properties are, therefore, obtained for highly excited levels. For the $\Omega=3 / 2$ and $J=3 / 2$ states of $\mathrm{LiO}$, Figure 2 (a) and Figure 2 (b) show the energy and $\langle\cos \theta\rangle$, respectively, as a function of $\mathrm{E}_{\mathrm{s}}$, for $\beta=0^{\circ}$ and 

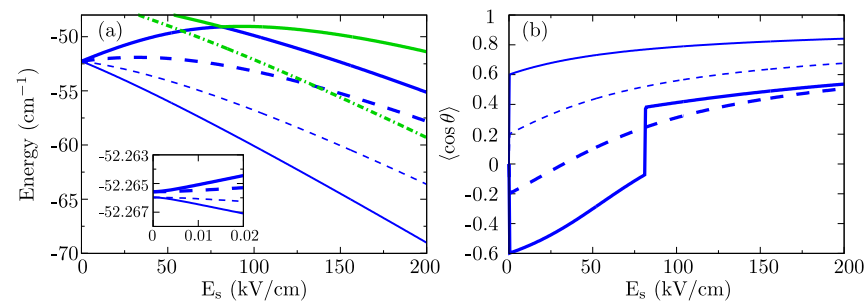

FIG. 2. For LiO, we present (a) the energy and (b) the expectation value $\left\langle\cos \theta_{\mathrm{s}}\right\rangle$ versus the electric field strength of the states $|3 / 2,3 / 2,3 / 2, \pm 1\rangle$ (blue thick and thin solid), and $|3 / 2,1 / 2,3 / 2, \pm 1\rangle$ (blue thick and thin dashed). Some states with $J=5 / 2$ are also plotted: $|5 / 2,5 / 2,3 / 2,1\rangle$ (green thick dot-dashed) and $|5 / 2,3 / 2,3 / 2,1\rangle$ (green thick solid). Due to the degeneracy in $\left|M_{J}\right|$, only those states with $M_{J}>0$ are presented. The inset in panel (a) shows the energy splitting for weak electric field. The field configuration is $\beta=0^{\circ}$ and $\mathrm{B}=0 \mathrm{~T}$.
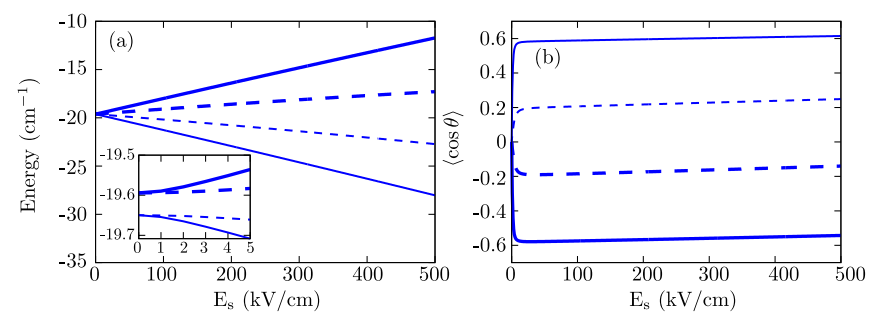

FIG. 3. For $\mathrm{OH}$, we present (a) the energy and (b) the expectation value $\left\langle\cos \theta_{\mathrm{s}}\right\rangle$ versus the electric field strength. The inset in panel (a) shows the energy splitting for weak electric field. The labeling of the states is done as in Figure 2 and due to the degeneracy in $\left|M_{J}\right|$, only those states with $M_{J}>0$ are plotted. The field configuration is $\beta=0^{\circ}$ and $\mathrm{B}=0 \mathrm{~T}$.

$\mathrm{B}=0 \mathrm{~T}$. The corresponding results for $\mathrm{OH}$ are presented in Figure 3 (a) and Figure 3 (b).

The Stark interaction (7) couples states with opposite parity, if $\beta=0^{\circ}, M_{J}$ is a good quantum number and the levels with $\pm M_{J}$ are still degenerate, although the field-free degeneracy in $M_{J}$ is broken. In the weak dcfield regime, the two $\Lambda$-doubling components are strongly coupled because they are energetically close. In addition, the coupling to the nearest rotational levels is rather weak because they are far apart in the spectrum. The $\Lambda$-doubling splitting within the $\Omega=3 / 2$ and $J=3 / 2$ manifold are $\sim 10^{-4} \mathrm{~cm}^{-1}$ and $\sim 5.6 \times 10^{-2} \mathrm{~cm}^{-1}$ for $\mathrm{LiO}$ and $\mathrm{OH}$, respectively; whereas they are separated by $5.96 \mathrm{~cm}^{-1}$ and $44.5 \mathrm{~cm}^{-1}$ to the levels with $\Omega=3 / 2$ and $J=5 / 2$. Thus, in the weak dc-field regime, the system could be described as a two state model formed by the even and odd parity levels with the same $M_{J}$. Only for very strong static electric fields, the couplings to the states within the next rotational manifold might become more important. Within this approximation, the Stark effect correction reads

$$
\Delta E= \pm \sqrt{\frac{E_{\Lambda}^{2}}{4}+\mu_{e f f}^{2} \mathrm{E}_{\mathrm{s}}^{2}}
$$

for the $s= \pm 1$ states with $E_{\Lambda}$ being the $\Lambda$-doublet splitting, and $\mu_{e f f}=-\mu\left|M_{J}\right| \Omega / J(J+1)$. The odd and even parity states are initially high- and low-field seekers, respectively. If the $\Lambda$-doublet splitting is small, even a very weak electric field might induce an efficient orientation, but the two states with different parity have their effective electric dipole moments pointing in opposite directions. For $\mathrm{LiO}$ and $\mathrm{E}_{\mathrm{s}}=20 \mathrm{~V} / \mathrm{cm},\langle\cos \theta\rangle= \pm 0.60$ and \pm 0.20 for the even and odd states with $\left|M_{J}\right|=3 / 2$ and $1 / 2$, respectively. For $\mathrm{OH}$, a stronger dc-field is needed to achieve a similar orientation, e. g., $\langle\cos \theta\rangle= \pm 0.57$ and \pm 0.19 for the even and odd states with $\left|M_{J}\right|=3 / 2$ and $1 / 2$, respectively, for $\mathrm{E}_{\mathrm{s}}=10 \mathrm{kV} / \mathrm{cm}$.

By further increasing $E_{s}$, the couplings to the next rotational manifold, and, therefore, the contribution of these states to the field-dressed dynamics should become more important. However, this is not the case for $\mathrm{OH}$, and these couplings are still small even for strong electric fields due to its large rotational constant. For all the $J=$ $3 / 2$ levels, the variation of $\left\langle\mathbf{J}^{2}\right\rangle$ compared to the field-free value is below $0.8 \%$ even for $\mathrm{E}_{\mathrm{s}}=500 \mathrm{kV} / \mathrm{cm}$. Then, the $\mathrm{OH}$ states keep their low- and high-field seeking character and their orientations vary smoothly, cf. Figure 3.

In contrast, these couplings to high $J$-value states leave their fingerprints in the $\mathrm{LiO}$ spectrum even at moderate electric field strength. To illustrate the hybridization of the total angular momentum in $\mathrm{LiO}$, we have plotted in Figure $4\left\langle\mathbf{J}^{2}\right\rangle$ versus $\mathrm{E}_{\mathrm{s}}$ for the $J=3 / 2$ and $J=5 / 2$ levels of ${ }^{2} \Pi_{3 / 2}$. For weak electric fields, $\left\langle\mathbf{J}^{2}\right\rangle$ presents a plateau-like behaviour around the fieldfree values $\left\langle\mathbf{J}^{2}\right\rangle=3.75$ and 8.75 for the $J=3 / 2$ and $J=5 / 2$ states, respectively. For the $|5 / 2, \pm 3 / 2,3 / 2,-1\rangle$ and $|5 / 2, \pm 1 / 2,3 / 2,-1\rangle$ levels, the coupling to states in the lowest rotational manifold is initially dominant, and $\left\langle\mathbf{J}^{2}\right\rangle$ decreases as $E_{\mathrm{s}}$ is increased, reaches a broad minimum increasing thereafter. For the rest, $\left\langle\mathbf{J}^{2}\right\rangle$ monotonically increases due to the couplings to states with higher field-free $J$-values. The $|3 / 2, \pm 3 / 2,3 / 2,1\rangle$ levels suffer an avoided crossing with $|5 / 2, \pm 3 / 2,3 / 2,1\rangle$ from the next rotational manifold for $\mathrm{E}_{\mathrm{s}} \approx 81.437 \mathrm{kV} / \mathrm{cm}$ of width $\Delta E=1.01 \times 10^{-3} \mathrm{~cm}^{-1}$, and they interchange their intrinsic character. Then, the $|3 / 2, \pm 3 / 2,3 / 2,1\rangle$ levels become oriented and acquire a high-field seeking character, see Figure 2. In the strong dc-field regime, all the $J=3 / 2$ states are high-field seekers, and their orientation monotonically increases as $\mathrm{E}_{\mathrm{s}}$ is increased. For $\mathrm{E}_{\mathrm{s}}=200 \mathrm{kV} / \mathrm{cm}$, the levels with $\left|M_{J}\right|=3 / 2$ and $1 / 2$ and $s=1$ have achieved a significant orientation with $\langle\cos \theta\rangle=0.84$ and 0.68 , respectively, whereas $\langle\cos \theta\rangle=$ 0.54 and 0.51 for the corresponding states with odd parity. They also show a strong hybridization of the angular motion, e. g., for $\mathrm{E}_{\mathrm{s}}=200 \mathrm{kV} / \mathrm{cm},\left\langle\mathbf{J}^{2}\right\rangle=5.11$ and 6.62 (8.37 and 11.18) for the odd (even) $\left|M_{J}\right|=3 / 2$ and $1 / 2$ states. 


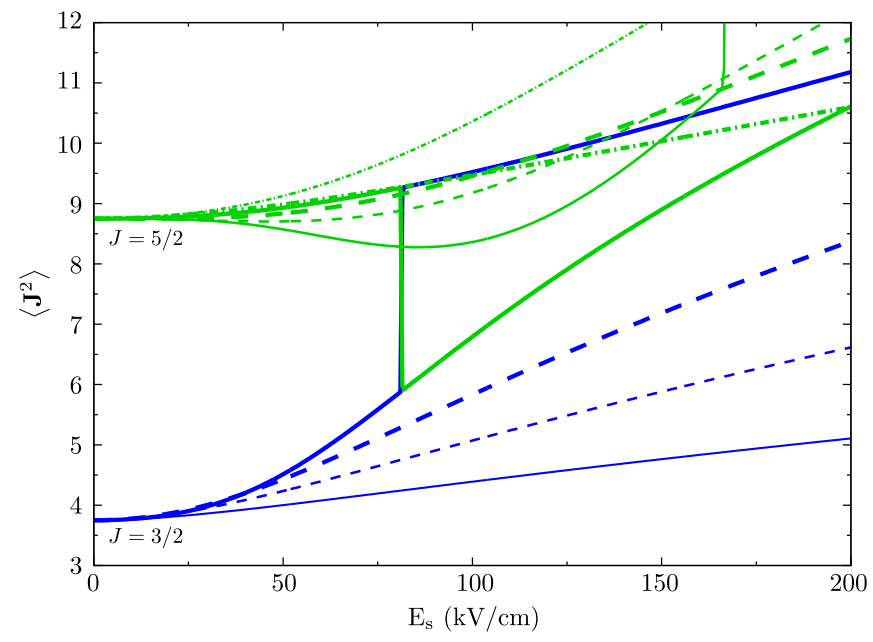

FIG. 4. For $\mathrm{LiO}$, we show the expectation value $\left\langle\mathbf{J}^{2}\right\rangle$ versus the electric field strength of the states $|3 / 2,3 / 2,3 / 2, \pm 1\rangle$ (blue thick and thin solid), $|3 / 2,1 / 2,3 / 2, \pm 1\rangle$ (blue thick and thin dashed), $|5 / 2,5 / 2,3 / 2, \pm 1\rangle$ (green thick and thin dot-shortdashed), $|5 / 2,3 / 2,3 / 2, \pm 1\rangle$ (green thick and thin solid), and $|5 / 2,1 / 2,3 / 2, \pm 1\rangle$ (green thick and thin dashed). Due to the degeneracy in $\left|M_{J}\right|$, only those states with $M_{J}>0$ are plotted. The field configuration is $\beta=0^{\circ}$ and $\mathrm{B}=0 \mathrm{~T}$.

\section{B. Influence of the magnetic field strength}

Here, we investigate the evolution of the energy as the magnetic field strength is increased. For the $\mathrm{OH}$ and $\mathrm{LiO}$ molecules, Figure 5 (a) and Figure 5 (b) show the energy of the $\Omega=3 / 2$ and $J=3 / 2$ states as a function of B with $\mathrm{E}_{\mathrm{s}}=0 \mathrm{~V} / \mathrm{cm}$ and $\beta=0^{\circ}$. In this field configuration, $M_{J}$ and $s$ are good quantum numbers. The states with $M_{J}<0(>0)$ are high- (low-) field seekers as B is varied, and the two components of a $\Lambda$-doublet run parallel. The $\mathrm{OH}$ molecule presents the linear Zeeman effect, i. e., the energy linearly depends on the magnetic field strength $\mathrm{B}$, and the states with different parity and $M_{J}$ suffer exact crossings, see inset in Figure 5, when B is increased. For $\mathrm{OH}$, the mixing with states of the neighbouring rotational manifold is negligible even at $\mathrm{B}=10 \mathrm{~T}$, where the variation of $\left\langle\mathbf{J}^{2}\right\rangle$ compared to its field-free value is below $1.2 \%$, and the alignment is also weakly affected.

Due to the small $\Lambda$-doubling splitting of $\mathrm{LiO}$, the states with even and odd parity are very close in energy and indistinguishable on the scale of Figure 5 (b). If the magnetic field is strong enough, the coupling to levels of the neighbouring $J$-manifold becomes more important, and the linear Zeeman behaviour is lost. The alignment $\left\langle\cos ^{2} \theta\right\rangle$ and hybridization of the angular motion $\left\langle\mathbf{J}^{2}\right\rangle$ are presented in Figure 6 (a) and (b), respectively. The alignment of the states with $M_{J}<0(>0)$ increases (decreases) as B is increased. Only the levels with $M_{J}=-3 / 2$ present moderate alignment with $\left\langle\cos ^{2} \theta\right\rangle>0.5$ for $\mathrm{B} \gtrsim 1.7 \mathrm{~T}$. As in the case of a static

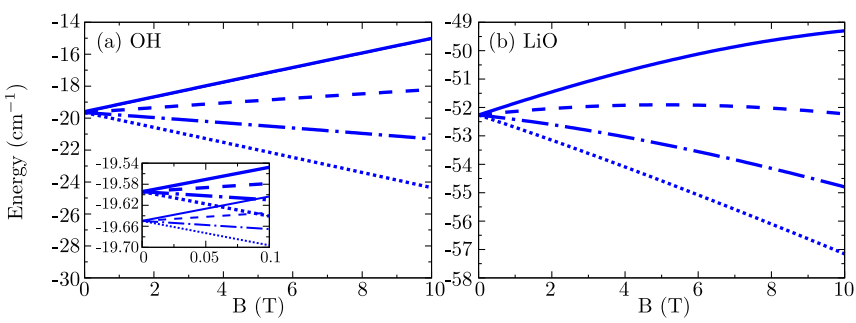

FIG. 5. For the $\Omega=3 / 2$ and $J=3 / 2$ states, we show their energy versus the magnetic field strength for (a) $\mathrm{OH}$ and (b) $\mathrm{LiO}$. The inset in panel (a) shows the energy splitting for weak magnetic field. The states are $|3 / 2,3 / 2,3 / 2, \pm 1\rangle$ (thick and thin solid), $|3 / 2,-3 / 2,3 / 2, \pm 1\rangle$ (thick and thin dotted), $|3 / 2,1 / 2,3 / 2, \pm 1\rangle$ (thick and thin dashed), and $|3 / 2,-1 / 2,3 / 2, \pm 1\rangle$ (thick and thin dot-dashed). Due to the small $\Lambda$-doubling the two states forming a $\Lambda$-doublet cannot be distinguished on the scale of this figure. The field configuration is $\beta=0^{\circ}$ and $\mathrm{E}_{\mathrm{s}}=0 \mathrm{~V} / \mathrm{cm}$.
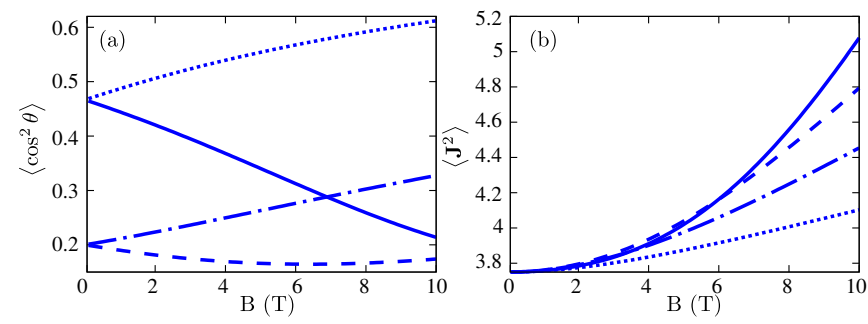

FIG. 6. For the $\Omega=3 / 2$ and $J=3 / 2$ states of $\mathrm{LiO}$, we show their expectation values (a) $\left\langle\cos ^{2} \theta_{\mathrm{s}}\right\rangle$ and (b) $\left\langle\mathbf{J}^{2}\right\rangle$ versus the magnetic field strength. The field configuration is $\beta=0^{\circ}$ and $\mathrm{E}_{\mathrm{s}}=0 \mathrm{~V} / \mathrm{cm}$. The labeling of the states is done as in Figure 5

electric field, $\left\langle\mathbf{J}^{2}\right\rangle$ monotonically increases with B.

\section{Influence of combined magnetic and electric fields}

The rotational dynamics is drastically modified when the molecules are exposed to combined magnetic and electric fields. The Zeeman and Stark interactions break different symmetries of the field-free Hamiltonian, and the order in which the fields are turned on determines the evolution of the field-dressed states. Indeed, their labels depend on the path followed on the parameter space, $\mathrm{E}_{\mathrm{s}}, \mathrm{B}$ and $\beta$, to reach a certain field configuration. This phenomenon, called monodromy, has been previously observed in diatomic and polyatomic molecules in external fields [32, 33]. In Figure 7] it is illustrated by the evolution of the $\Omega=3 / 2$ and $J=3 / 2$ states of $\mathrm{OH}$ increasing first the electric field and then the magnetic one (left panel) or vice versa (right panel) with $\beta=30^{\circ}$.

These two pathways through the parameter space lead to different state labels at the final point. The main rea- 


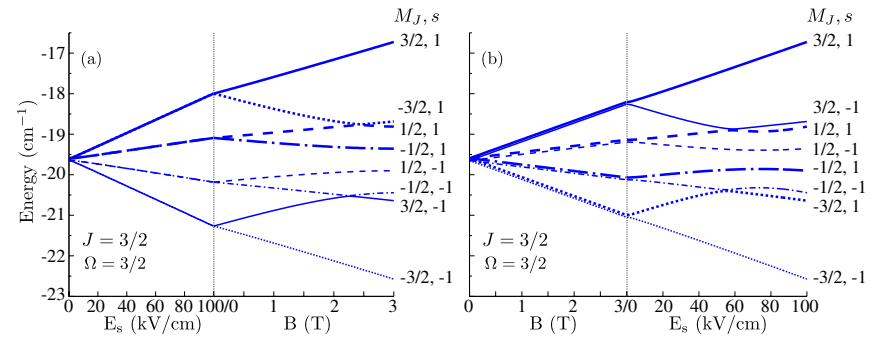

FIG. 7. The $J=3 / 2$ manifold of ${ }^{2} \Pi_{3 / 2}$ is presented at intermediate field strengths. The relative angle between the fields is $\beta=30^{\circ}$. The labels of the states are put according to the adiabatic following.

son is that, as indicated above, by modifying the field configuration we change the symmetries of the system and on different parameter pathways the symmetries are broken in different order. The energetic ordering depends on which field is present at first. If the electric field is turned on first, the parity in the field-free limit determines the low or high-field seeking character of a level, see Figure 7 (a), whereas in the case of a magnetic field, it is the sign of $M_{J}$, cf. Figure 7 (b). It shall be noted that the electric field immediately mixes states of different parity. So the label $s$ is given according to adiabatic following and no longer has the meaning of the parity of the eigenstate but indicates if a state is oriented $(s=-1)$ or anitoriented $(s=1)$. As the second field is switched on, the previous energetic ordering of the states is kept since there are no more good quantum numbers and all crossings encountered in the spectrum are avoided, except for the case $\beta=90^{\circ}$. This non-uniqueness of the state labeling is typical for systems which cannot be described using a single set of irreducible representations in the whole parameter space.

\section{Constant magnetic field strength and increasing electric field strength}

Now, we consider a field configuration in which after turning on a magnetic field of $\mathrm{B}=1 \mathrm{~T}$, the electric field is switched on forming an angle $\beta$ with the LFF $Z$-axis. For $\mathrm{LiO}$ and $\mathrm{OH}$, the variation of the energies of the states with $\Omega=3 / 2$ and $J=3 / 2$ as a function of $\mathrm{E}_{\mathrm{s}}$ is presented in Figure 8 (a), (b), (c) and (d) and Figure 9 (a), (b), (c) and (d), for the inclination angles $\beta=0^{\circ}, 30^{\circ}, 60^{\circ}$ and $90^{\circ}$, respectively. The corresponding results for the orientation cosines $\left\langle\cos \theta_{\mathrm{s}}\right\rangle$ are presented in Figure 10 and Figure 11.

Let us start analyzing the results for the parallelfield configuration. In this case, $M_{J}$ is still a good quantum number, and within this manifold the states suffer exact crossings. At $\mathrm{E}_{\mathrm{s}}=0 \mathrm{~V} / \mathrm{cm}$ and $\mathrm{B}=$ $1 \mathrm{~T}$, the $M_{J}$-degeneracy is already lifted and the 8 states are identified on the $\mathrm{OH}$ spectrum, whereas for
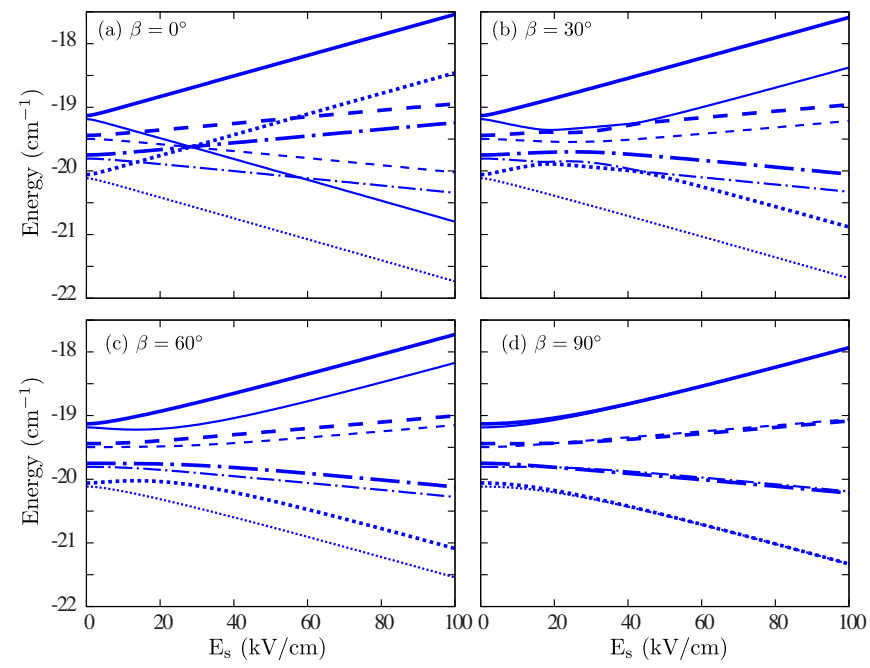

FIG. 8. For the $\Omega=3 / 2$ and $J=3 / 2$ states of $\mathrm{OH}$, we present the energy versus the electric field strength for (a) $\beta=0^{\circ}$, (b) $\beta=30^{\circ}$, (b) $\beta=60^{\circ}$ and (c) $\beta=90^{\circ}$, with $\mathrm{B}=1 \mathrm{~T}$. The labeling of the states is done as in Figure 5

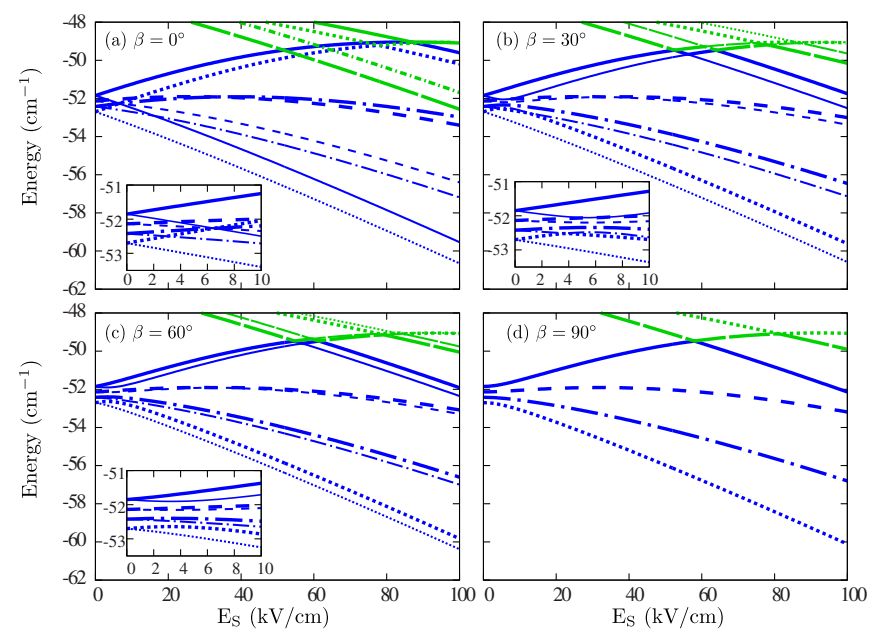

FIG. 9. Same as Figure 8 but for LiO. The insets show the weak electric field regime. Some states from the rotational manifold $J=5 / 2$ are included: $|5 / 2,5 / 2,3 / 2,1\rangle$ (green thick dot-short-dashed), $|5 / 2,-5 / 2,3 / 2, \pm 1\rangle$ (green thick and thin long-dashed), $|5 / 2,3 / 2,3 / 2,1\rangle$ (green thick solid), and $|5 / 2,-3 / 2,3 / 2, \pm 1\rangle$ (green thick and thin dotted).

$\mathrm{LiO}$, the $\Lambda$-doubling is so small that the even and odd parity states are still quasidegenerate. $A_{s} E_{s}$ is increased the odd (even) parity states become high- (low-) field seekers. The levels $|3 / 2,-3 / 2,3 / 2,1\rangle,|3 / 2,-1 / 2,3 / 2,1\rangle$, $|3 / 2,1 / 2,3 / 2,-1\rangle$ and $|3 / 2,3 / 2,3 / 2,-1\rangle$ have the same energy for $\mathrm{E}_{\mathrm{s}} \approx 28 \mathrm{kV} / \mathrm{cm}$ and $\mathrm{E}_{\mathrm{s}} \approx 6.4 \mathrm{kV} / \mathrm{cm}$ for $\mathrm{OH}$ and $\mathrm{LiO}$ respectively. For $\mathrm{LiO}$, we find an avoided crossing among the levels $|3 / 2,-3 / 2,3 / 2,1\rangle$ and $|5 / 2,-3 / 2,3 / 2,1\rangle$ at $\mathrm{E}_{\mathrm{s}} \approx 77.782 \mathrm{kV} / \mathrm{cm}$ of width $\Delta E=8.5 \times 10^{-4} \mathrm{~cm}^{-1}$, a second one is encounter between the states $|3 / 2,3 / 2,3 / 2,1\rangle$ 
and $|5 / 2,3 / 2,3 / 2,1\rangle$ at $\mathrm{E}_{\mathrm{s}} \approx 86.7827 \mathrm{kV} / \mathrm{cm}$ of width $\Delta E=1.15 \times 10^{-3} \mathrm{~cm}^{-1}$. For this system, all the states are high-field seekers in the strong field regime.

For non-parallel fields $\beta \neq 90^{\circ}$, there are no symmetries left on the rigid-rotor Hamiltonian. The real crossings appearing for $\beta=0^{\circ}$ become avoided, in particular, the states $|3 / 2,-3 / 2,3 / 2,1\rangle$ and $|3 / 2,-1 / 2,3 / 2,-1\rangle$ suffer two consecutive avoided crossings at $\mathrm{E}_{\mathrm{s}} \approx 3.7835 \mathrm{kV} / \mathrm{cm}$ with $\Delta E=2.2 \times 10^{-4} \mathrm{~cm}^{-1}$ and $\mathrm{E}_{\mathrm{s}} \approx 12.350 \mathrm{kV} / \mathrm{cm}$ with $\Delta E=7.2 \times 10^{-5} \mathrm{~cm}^{-1}$. Analogously, the levels $|3 / 2,3 / 2,3 / 2,-1\rangle$ and $|3 / 2,1 / 2,3 / 2,1\rangle$ undergo two avoided intersections. The positions of these avoided intersections is shifted as $\beta$ is varied, and their widths increase as $\beta$ increases and approaches $90^{\circ}$. In the strong electric field regime, the energies of the states having the same field-free $M_{J}$ run parallel as $\mathrm{E}_{\mathrm{s}}$ is augmented. For perpendicular fields, the Hamiltonian is invariant under the reflection in the plane perpendicular to the magnetic field. When the electric field is strong enough to overcome the $\Lambda$-doublet splitting, then, the levels having the same field-free $\left|M_{J}\right|$ form pairs of quasidegenerate states. For $\mathrm{OH}$, the energy gap within these doublets decreases as $\mathrm{E}_{\mathrm{s}}$ is increased. Whereas for LiO, again due to the small $\Lambda$-doubling, the two states are quasidegenerate even for weak dc fields.

In the parallel field configuration, the states with fieldfree odd and even parity are oriented and antioriented, respectively, see Figure 10 (a) and Figure 11(a), but the additional magnetic field does not modify significantly their absolute orientation $\left|\left\langle\cos \theta_{\mathrm{s}}\right\rangle\right|$. For $\mathrm{LiO},\left|\left\langle\cos \theta_{\mathrm{s}}\right\rangle\right|$ is larger for the state having a negative field-free $M_{J}$. If the two fields are tilted, the presence of new avoided crossings modifies the directional properties of the states, and $\left\langle\cos \theta_{\mathrm{s}}\right\rangle$ changes abruptly in the proximity of these irregular regions. Thus, for small variations of the electric field strength, a molecule prepared in the anti-oriented state would flip its dipole moment and turn into oriented. Such an electric field steered dipole switcher could have applications in tailoring the interactions between polar molecules. By ramping the electric field strength through these crossings at different speeds, the Landau-Zener dynamics could be studied and the Landau-Zener tunneling probability measured. For a strong enough electric field with $\beta>0^{\circ}$, the pairs of states $\left|3 / 2, M_{J}, 3 / 2, \pm 1\right\rangle$ have very close orientation. All the $J=3 / 2$ levels of $\mathrm{LiO}$ are orientated parallel to the field in the strong dc-field regime, and their $\left\langle\cos \theta_{\mathrm{s}}\right\rangle$ increases as $\mathrm{E}_{\mathrm{s}}$ is increased.

To illustrate the impact of an additional magnetic field on the hybridization of the angular motion of $\mathrm{LiO}$, we have plotted the expectation value $\left\langle\mathbf{J}^{2}\right\rangle$ of $|3 / 2,-1 / 2,3 / 2,1\rangle$ and $|3 / 2,3 / 2,3 / 2,1\rangle$ as a function of $E_{\mathrm{s}}$ in Figure 12 (a) and Figure 12 (b), respectively. There we provide a comparison between the results of the field configurations $\mathrm{B}=1 \mathrm{~T}$ with $\beta=0^{\circ}, 30^{\circ} 60^{\circ}$ and $90^{\circ}$ with those obtained with only an electric field. As indicated above, the level $|3 / 2,-1 / 2,3 / 2,1\rangle$ suffer real crossings with neighbouring levels for $\beta=0^{\circ}$; and due to the level reordering it suffers an avoided crossings for $\beta>0^{\circ}$ with

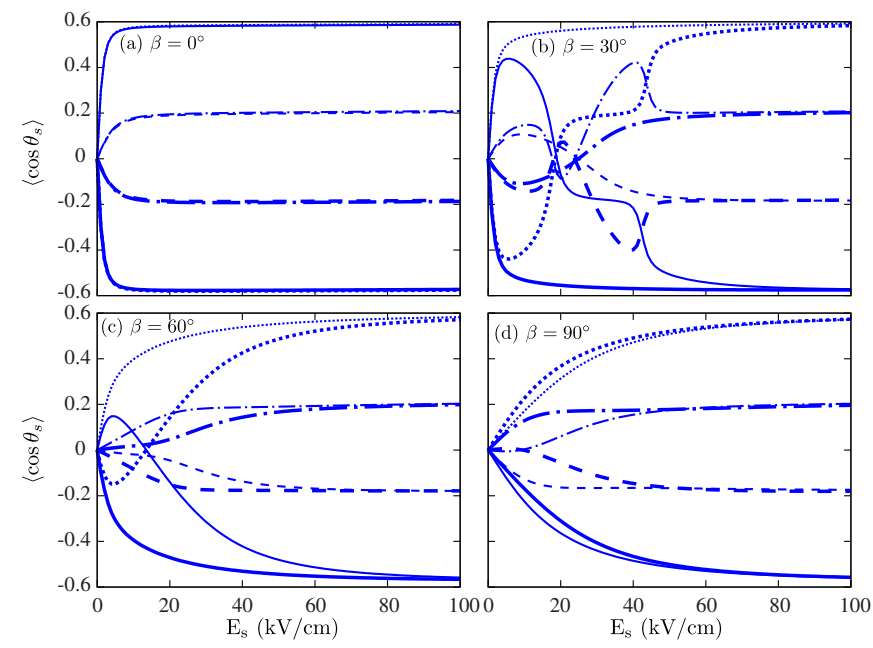

FIG. 10. For the $\Omega=3 / 2$ and $J=3 / 2$ states of $\mathrm{OH}$, the expectation value $\left\langle\cos \theta_{\mathrm{s}}\right\rangle$ is shown versus the electric field strength for (a) $\beta=0^{\circ}$, (b) $\beta=30^{\circ}$, (c) $\beta=60^{\circ}$ and (d) $\beta=90^{\circ}$, with $\mathrm{B}=1 \mathrm{~T}$. The labeling of the states is done as in Figure 5 .

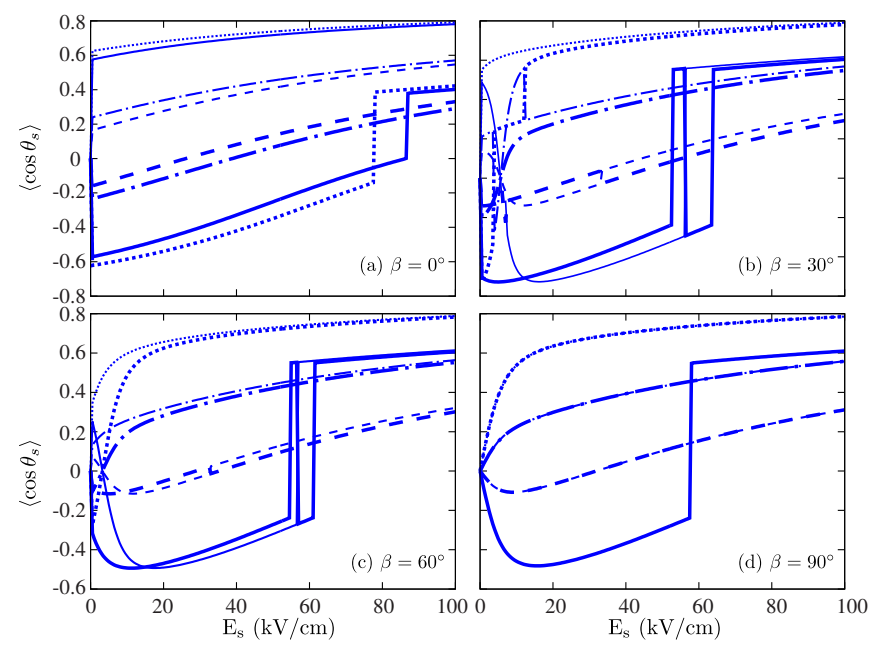

FIG. 11. Same as Figure 10 but for LiO.

a state in the same $J=3 / 2$ manifold. The effect of this avoided crossing on $\left\langle\mathbf{J}^{2}\right\rangle$ is very small, because both levels have the same field-free value of $\left\langle\mathbf{J}^{2}\right\rangle$. Thus, independently of $\beta$, its $\left\langle\mathbf{J}^{2}\right\rangle$ shows a smooth and increasing behaviour as $\mathrm{E}_{\mathrm{s}}$ is increased. Furthermore, independently of $E_{s},\left\langle\mathbf{J}^{2}\right\rangle$ achieves the largest value in the absence of the magnetic field. This is explained by the increase of the energy separation of this state from the neighbouring $J=5 / 2$ levels for $\beta>0^{\circ}$, which results in a reduction of the hybridization of the angular motion, and, therefore, of $\left\langle\mathbf{J}^{2}\right\rangle$. A different behaviour is observed for the state $|3 / 2,3 / 2,3 / 2,1\rangle$. For all these field configurations, $|3 / 2,3 / 2,3 / 2,1\rangle$ has the larger energy within the $J=3 / 2$ manifold of ${ }^{2} \Pi_{3 / 2}$ and shows a low-field seeking charac- 


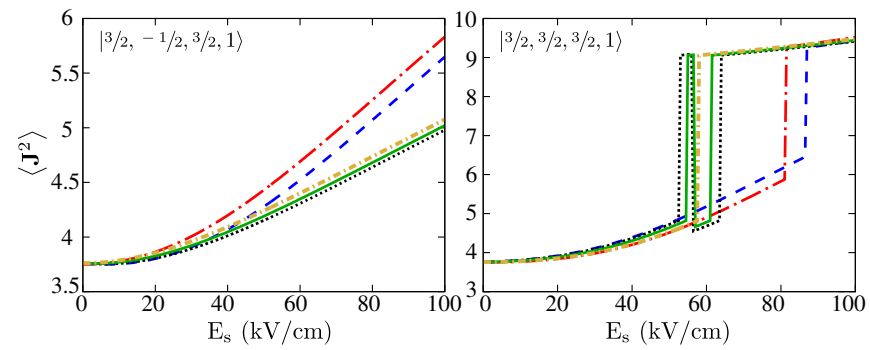

FIG. 12. For LiO, we show the expectation value $\left\langle\mathbf{J}^{2}\right\rangle$ of the states $|3 / 2,-1 / 2,3 / 2,1\rangle$ and $|3 / 2,3 / 2,3 / 2,1\rangle$ versus the electric field strength for $\mathrm{B}=0 \mathrm{~T}$ (dot-long-dashed), and $\mathrm{B}=1 \mathrm{~T}$ with $\beta=0^{\circ}$ (dashed), $\beta=30^{\circ}$ (dotted), $\beta=60^{\circ}$ (solid), and $\beta=90^{\circ}$ (dot-short-dashed).

ter when the dc field is turned on. As a consequence, it suffers several avoided crossings with high-field seekers from the $J=5 / 2$ manifold of ${ }^{2} \Pi_{3 / 2}$, which are reflected as abrupt changes of $\left\langle\mathbf{J}^{2}\right\rangle$ when $\mathrm{E}_{\mathrm{S}}$ is varied. Note that for $\beta=30^{\circ}$ and $60^{\circ}$, this state undergoes two consecutive avoided crossings. A third kind of behaviour is encountered: For levels such as the rotational ground state $|3 / 2,-3 / 2,3 / 2,-1\rangle$, the additional magnetic field provokes only minor variations on $\left\langle\mathbf{J}^{2}\right\rangle$.

The behavior for a weak electric field illustrates nicely an interesting feature of open shell states compared to $\Sigma$ electronic states: In the presence of only a magnetic field, the ground state $|3 / 2,3 / 2,3 / 2,-1\rangle$ is separated from $|3 / 2,3 / 2,3 / 2,1\rangle$ by the $\Lambda$-doubling splitting. By switching on an electric field, these two almost degenerate states mix immediately, and the oriented superposition becomes the ground state while the anti-oriented one increases in energy with $\mathrm{E}_{\mathrm{s}}$ (see Figure 8 and Figure 9). The two states of this doublet are indeed strongly oriented and antioriented, respectively, before they start to interact with other states. If one could prepare the ground state in such a field configuration, this amounts to a two-level molecule with an oriented and an antioriented component. If the dc field is turned on slowly, the molecule adiabatically follows the oriented ground state. Whereas, population could be transferred to the antioriented level if the switching on is done fast enough. In a molecular ensemble, where dipole-dipole interaction has to be taken into account, this allows one to access different phases of the many-body (spin-) system by tuning the field parameters or the speed of the field ramps. The implementation of spin Hamiltonians with a high degree of control over the system parameters may be achieved this way. Also, field parameters could be used to shape the intermolecular interactions themselves $34-36$.
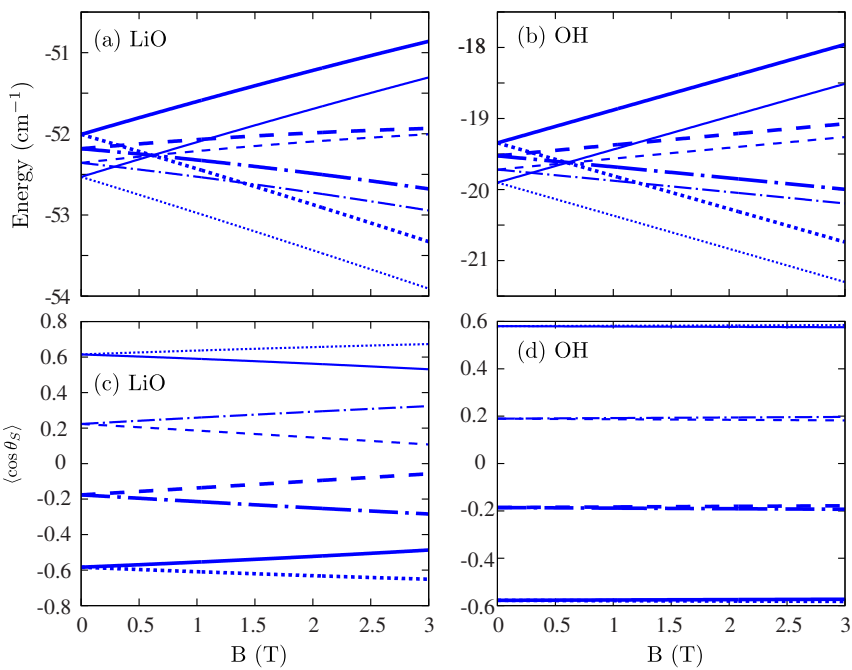

FIG. 13. For the $J=3 / 2$ levels of the ${ }^{2} \Pi_{3 / 2}$ state of $\mathrm{LiO}$ and $\mathrm{OH}$, energy and expectation value $\left\langle\cos \theta_{\mathrm{s}}\right\rangle$ as a function of $\mathrm{B}$ for the field configuration $\mathrm{E}_{\mathrm{s}}=4 \mathrm{kV} / \mathrm{cm}$ and $\mathrm{E}_{\mathrm{s}}=17 \mathrm{kV} / \mathrm{cm}$, respectively and $\beta=0^{\circ}$. The labeling of the states is the same as in Figure 5.

\section{Constant electric field strength and increasing magnetic field strength}

Here we analyze the impact of increasing the magnetic field strength, when the system is also exposed to an electric field, which is turned on first. For the $J=3 / 2$ levels of the $\mathrm{LiO}$ and $\mathrm{OH}^{2} \Pi_{3 / 2}$ electronic states, Figure 13 and Figure 14 display the energies and orientation cosines as $\mathrm{B}$ is increased for $\beta=0^{\circ}$ and $\beta=30^{\circ}$, respectively. For $\mathrm{LiO}$ and $\mathrm{OH}$, the dc field strengths are fixed to $\mathrm{E}_{\mathrm{s}}=4 \mathrm{kV} / \mathrm{cm}$ and $\mathrm{E}_{\mathrm{s}}=17 \mathrm{kV} / \mathrm{cm}$, respectively, so that at $\mathrm{B}=1 \mathrm{~T}$ and $\beta=0^{\circ}$ the Zeeman and Stark interactions are of the same order of magnitude.

For parallel fields, the field-free states with $s=1$ show a high-field seeking behaviour as B is increased, whereas those with $s=-1$ are low-field seekers. Since $M_{J}$ is still a good quantum number, we encounter several exact crossings among these levels, whose positions are different for $\mathrm{LiO}$ and $\mathrm{OH}$. The directional properties of $\mathrm{OH}$ are not affected by the additional magnetic field. In contrast, $\left\langle\cos \theta_{\mathrm{s}}\right\rangle$ increases (decreases) for the levels of $\mathrm{LiO}$ with field-free even (odd) parity as B is enhanced.

When the fields are tilted, all the real intersections become avoided, and the complexity of the field-dressed spectrum increases significantly. The widths of these avoided crossings increases as $\beta$ is increased toward $90^{\circ}$. Compared to $\mathrm{LiO}$, the avoided crossings in $\mathrm{OH}$ are broader. For instance, the $\mathrm{OH}$ levels $|3 / 2,+3 / 2,3 / 2,-1\rangle$ and $|3 / 2,-1 / 2,3 / 2,-1\rangle$ suffer an avoided crossing at $\mathrm{B}=$ $0.3778 \mathrm{~T}$ with $\Delta E=1.386 \times 10^{-2} \mathrm{~cm}^{-1}$, whereas for $\mathrm{LiO}$ it occurs at $\mathrm{B}=0.3750 \mathrm{~T}$ with $\Delta E=$ $9.4 \times 10^{-5} \mathrm{~cm}^{-1}$. The states $|3 / 2,-3 / 2,3 / 2,-1\rangle$ and $|3 / 2,3 / 2,3 / 2,1\rangle$ do not suffer any avoided crossing, and 

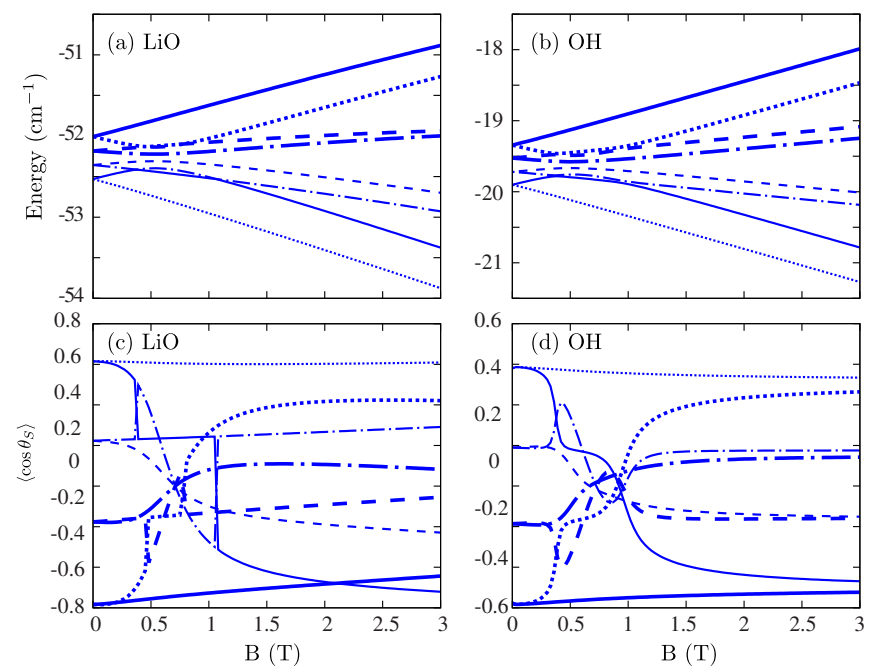

FIG. 14. Same as Figure 13 but for $\beta=30^{\circ}$

their orientation smoothly varies as B is increased. Compared to the parallel field configuration, their absolute orientation $\left|\left\langle\cos \theta_{\mathrm{s}}\right\rangle\right|$ is reduced. For the remainder of the states, $\left\langle\cos \theta_{\mathrm{s}}\right\rangle$ changes abruptly in the proximity of the avoided crossing. Once the magnetic field is strong enough their $\left\langle\cos \theta_{\mathrm{s}}\right\rangle$ also shows a smooth behaviour as a function of $\mathrm{B}$.

\section{Influence of the inclination of the fields}

In this section, we investigate the impact of the inclination angle on the rotational dynamics. For the lowest lying rotational manifold of $\mathrm{LiO}$, i. e., the $J=3 / 2$ levels of ${ }^{2} \Pi_{3 / 2}$, we present in Figure 15 (a) and Figure 15 (b) the energy and orientation of the dipole moment with respect to the electric field, $\left\langle\cos \theta_{\mathrm{s}}\right\rangle$, respectively, as a function of $\beta$ with $\mathrm{E}_{\mathrm{s}}=10 \mathrm{kV} / \mathrm{cm}$ and $\mathrm{B}=2 \mathrm{~T}$. In this regime of field strengths, only states within a rotational manifold suffer avoided crossings with each other. The admixture of states having $J=5 / 2$ is very small. For $\beta=0^{\circ}$, the degeneracy of the states is lifted, with $M_{J}$ being still a good quantum number. As $\beta$ is varied the symmetries of the system are reduced, and for orthogonal fields, pairs of quasidegenerate states are formed, which is a sign for the emergence of a new symmetry.

The states $|3 / 2,-3 / 2,3 / 2,-1\rangle, \quad|3 / 2,3 / 2,3 / 2,1\rangle$, $|3 / 2,-1 / 2,3 / 2,1\rangle$, and $|3 / 2,1 / 2,3 / 2,-1\rangle$ do not suffer any avoided crossings as $\beta$ is varied, and their orientation changes smoothly. The state $|3 / 2,-3 / 2,3 / 2,-1\rangle$ presents the larger orientation, and $\left\langle\cos \theta_{\mathrm{s}}\right\rangle$ is reduced from 0.67 for $\beta=0^{\circ}$ till 0.40 for $\beta=90^{\circ}$.

The pair $|3 / 2,-3 / 2,3 / 2,1\rangle$ and $|3 / 2,-1 / 2,3 / 2,-1\rangle$ suffer an avoided crossing, and around this irregular region their orientation changes abruptly, analogously for the $|3 / 2,3 / 2,3 / 2,-1\rangle$ and $|3 / 2,1 / 2,3 / 2,1\rangle$ levels. After these avoided crossings, their directional properties evolve
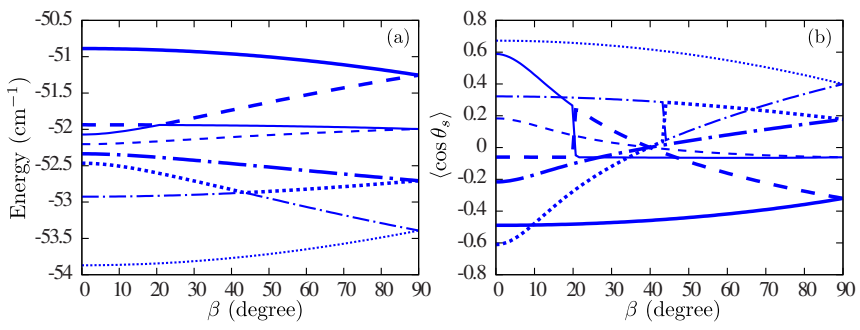

FIG. 15. For the $J=3 / 2$ levels of the ${ }^{2} \Pi_{3 / 2}$ state of LiO, (a) the energy and (b) the expectation value $\left\langle\cos \theta_{\mathrm{s}}\right\rangle$ are plotted as a function of the inclination angle $\beta$. The field configuration is $\mathrm{B}=2 \mathrm{~T}$ and $\mathrm{E}_{\mathrm{s}}=10 \mathrm{kV} / \mathrm{cm}$. The states are labeled as in Figure 5 .

smoothly as $\beta$ is enhanced towards $90^{\circ}$. For $\beta=39.5^{\circ}$, the states $|3 / 2,-3 / 2,3 / 2,1\rangle,|3 / 2,-1 / 2,3 / 2,1\rangle,|3 / 2,1 / 2,3 / 2,1\rangle$, and $|3 / 2,1 / 2,3 / 2,-1\rangle$ do not show any orientation.

\section{A toy molecular system}

In this section, we explore the possibility of using external fields to couple rotational levels from the two fine structure components of a ${ }^{2} \Pi$ electronic state. Our aim is to investigate field-induced couplings between spin degrees of freedom and the molecular rotation. We are interested in predicting general properties and principal effects with the focus on their understanding, thus we do not address a specific molecule but use a toy system. This molecular model has been constructed using the $\mathrm{OH}$ molecule as a prototype: its rotational and spin-orbit constants have been reduced by a factor of 10 , whereas the spin-rotation and $\Lambda$-doubling parameters by a factor of 100 . We have used this ratio based on the simple pure precession hypothesis to estimate the $\Lambda$-doublet parameter $p=4 A B / \Delta E$, where $\Delta E$ is the energy separation between the ${ }^{2} \Pi$ electronic ground state and the ${ }^{2} \Sigma$ lowest excited electronic state. The dipole moment has been fixed to $\mu=2 \mathrm{D}$. Note that both, increasing the dipole moment or decreasing the rotational or spin-orbit constant, lead to the appearance of crossings at lower fields.

For this system, the field-free spectrum is similar to the $\mathrm{OH}$ energy structure presented in Figure 1, but the energy scales have been reduced so that the coupling between different degrees of freedom can be achieved at lower field strengths. In the ${ }^{2} \Pi_{3 / 2}$ fine structure component, the $J=3 / 2$ and $5 / 2$ rotational bands are separated by $8.4 \mathrm{~cm}^{-1}$, and they have $\Lambda$-doublet splittings of $5.6 \times 10^{-4} \mathrm{~cm}^{-1}$ and $2 \times 10^{-3} \mathrm{~cm}^{-1}$, respectively. The ground state of ${ }^{2} \Pi_{1 / 2}$ lies $12.6 \mathrm{~cm}^{-1}$ and $4.2 \mathrm{~cm}^{-1}$ above the $J=3 / 2$ and $J=5 / 2$ levels of ${ }^{2} \Pi_{3 / 2}$, respectively, and has a $\Lambda$-doublet splitting of $1.6 \times 10^{-3} \mathrm{~cm}^{-1}$.

Figure 16 (a) displays the energies for the levels with $J=3 / 2$ and $J=5 / 2$ of ${ }^{2} \Pi_{3 / 2}$ and $J=1 / 2$ of ${ }^{2} \Pi_{1 / 2}$ as a function of the electric strength. When the $\mathrm{dc}$ field is turned on, the levels in a $J$-manifold are driven 

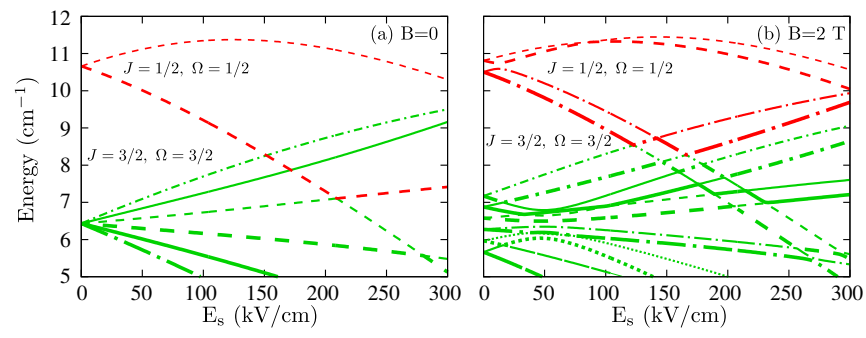

FIG. 16. For the toy system, we show the energy of the states with $J=5 / 2$ (green) from ${ }^{2} \Pi_{3 / 2}$ and $J=1 / 2$ (red) from ${ }^{2} \Pi_{1 / 2}$ as a function of the dc field strength $\mathrm{E}_{\mathrm{s}}$ for the field configuration (a) $\mathrm{B}=0 \mathrm{~T}$ and $\beta=0^{\circ}$ and (b) $\mathrm{B}=2 \mathrm{~T}$ and $\beta=30^{\circ}$. The labels of states are: $M_{J}=1 / 2$ and $s= \pm 1$ thick and thin dashed, $M_{J}=-1 / 2$ and $s= \pm 1$ thick and thin dot-dashed, $M_{J}=3 / 2$ and $s= \pm 1$ thick and thin solid, $M_{J}=-3 / 2$ and $s= \pm 1$ thick and thin dotted, $M_{J}=5 / 2$ and $s= \pm 1$ thick and thin dot-short-dashed, and $M_{J}=-5 / 2$ and $s= \pm 1$ thick and thin long-dashed.

apart in pairs having the same $\left|M_{J}\right|$ and depending on the field-free parity they have a high- or low-field seeking character. The $|1 / 2, \pm 1 / 2,1 / 2,1\rangle$ levels are high-field seekers, then, when $\mathrm{E}_{\mathrm{s}}$ is increased they will encounter the low-field seeking states from the $J=5 / 2$ manifold of ${ }^{2} \Pi_{3 / 2}$. Indeed, $|1 / 2, \pm 1 / 2,1 / 2,1\rangle$ undergoes exact crossings with $|5 / 2, \pm 5 / 2,3 / 2,-1\rangle$ and $|5 / 2, \pm 3 / 2,3 / 2,-1\rangle$ for $\mathrm{E}_{\mathrm{s}}=151.698 \mathrm{kV} / \mathrm{cm}$ and $\mathrm{E}_{\mathrm{s}}=171.718 \mathrm{kV} / \mathrm{cm}$, respectively. In addition, it suffers a very narrow avoided crossing with $|5 / 2, \pm 1 / 2,3 / 2,-1\rangle$ for $\mathrm{E}_{\mathrm{s}} \approx 209.22 \mathrm{kV} / \mathrm{cm}$ of width $\Delta E=2.1 \times 10^{-5} \mathrm{~cm}^{-1}$, and through it these levels interchange their intrinsic character. At these irregular regions, the dc field induces a strong mixing and interaction between the rotational and spin degrees of freedom. Thus, we encounter interactions between states in different rotational manifolds of given fine structure components, but also among levels in ${ }^{2} \Pi_{3 / 2}$ and ${ }^{2} \Pi_{1 / 2}$.

The results for this molecule exposed to an additional magnetic field of $2 \mathrm{~T}$ forming an angle $\beta=30^{\circ}$ with the dc field are presented in Figure 16 (b). Now, $M_{J}$ is not a good quantum number, the $\left|M_{J}\right|$-degeneracy is lifted and the real intersections among levels appearing in an electric field become avoided. Thus, the first avoided crossing between ${ }^{2} \Pi_{3 / 2}$ and ${ }^{2} \Pi_{1 / 2}$ states takes place at a lower electric field strength $\mathrm{E}_{\mathrm{s}}$. Indeed, the states $|1 / 2,-1 / 2,1 / 2,1\rangle$ and $|5 / 2,5 / 2,3 / 2,-1\rangle$ suffer an avoided intersection for $\mathrm{E}_{\mathrm{s}} \approx 122.898 \mathrm{kV} / \mathrm{cm}$, and energy width $2.96 \times 10^{-4} \mathrm{~cm}^{-1}$. Thus the effects due to the mixing of different fine structure components come into reach under experimentally realistic conditions. By further increasing $\mathrm{E}_{\mathrm{s}}$, we encounter a cascade of avoided crossings within the $J=5 / 2$ rotational manifold of ${ }^{2} \Pi_{3 / 2}$. We have identified the avoided crossing equivalent to the one at $209.22 \mathrm{kV} / \mathrm{cm}$ in the absence of the magnetic field. It now takes place between the states $|1 / 2,-1 / 2,1 / 2,1\rangle$ and $|5 / 2,1 / 2,3 / 2,1\rangle$ for $\mathrm{E}_{\mathrm{s}}=204.474 \mathrm{kV} / \mathrm{cm}$ and has a larger energy gap $\Delta E=1.4 \times 10^{-4} \mathrm{~cm}^{-1}$.

\section{CONCLUSIONS}

We have investigated the impact of combined electric and magnetic fields on the rotational spectrum of open shell diatomic molecules in a ${ }^{2} \Pi$ electronic state. This study has been performed within the rigid rotor approximation, including the fine-structure interactions and the $\Lambda$-doubling effects. For several field configurations, the richness and variety of the field-dressed rotational dynamics has been illustrated by analyzing the energies, the directional properties, and the hybridization of the angular motion.

Considering several field regimes, we have explored the role of the different interactions, and the possibility of inducing coupling between the different degrees of freedom by using the external fields. Due to the large rotational constant of the $\mathrm{OH}$ radical, we have shown that for the $J=3 / 2$ states of ${ }^{2} \Pi_{3 / 2}$, the field-dressed dynamics takes place within this manifold even for strong external fields, and the contribution of levels with higher $J$ is negligible. In contrast, we have proven that strong electric or magnetic fields induce coupling between states of different rotational manifolds of ${ }^{2} \Pi_{3 / 2}$ in the $\mathrm{LiO}$ spectrum. Finally, we have considered a realistic model system and demonstrated the feasibility of inducing coupling between rotational levels of the ${ }^{2} \Pi_{1 / 2}$ and ${ }^{2} \Pi_{3 / 2}$ electronic states by means of external fields.

In all these regimes, the complexity of the field-dressed spectrum is characterized by the amount of avoided crossings that the states suffer in the field-dressed dynamics. Around them, the fields become control knobs that could be exploited to tailor the interactions between polar molecules and influence their rotational dynamics. Indeed, by tuning the field parameters one can find configurations where either the dipole moment and orientation are flipped or the electronic spin interchanged. Then, Landau-Zener tunnelling between these states could be observed if the corresponding field strength is changed fast enough around the crossing region, and Rabi oscillations could be induced. Here, we have shown that these phenomena might appear at field strengths which are well within experimental reach.

\section{ACKNOWLEDGMENTS}

Financial support by the Spanish project FIS201124540 (MICINN), the Grants P11-FQM-7276 and FQM4643 (Junta de Andalucía), and the Andalusian research group FQM-207 is gratefully appreciated. J.J.O. acknowledges the support of ME under the program FPU.
[1] M. Zeppenfeld, B.G.U. Englert, R. Glöckner, A. Prehn, M. Mielenz, C. Sommer, L.D. van Buuren, M. Motsch and R. G., Nature 491, 570 (2012). 
[2] B.K. Stuhl, M.T. Hummon, M. Yeo, G. Quéméner, J.L. Bohn and J. Ye (2012), arXiv:1209.6343v1.

[3] M. Schnell and G. Meijer, Angew. Chem. Int. Ed. 48, 6010 (2009).

[4] K.K. Ni, S. Ospelkaus, M.H.G. de Miranda, A. Pe'er, B. Neyenhuis, J.J. Zirbel, S. Kotochigova, P.S. Julienne, D.S. Jin and J. Ye, Science 322, 231 (2008).

[5] M.T. Hummon, M. Yeo, B.K. Stuhl, A.L. Collopy, Y. Xia and J. Ye (2012), arXiv:1209.4069v1.

[6] S. Stellmer, B. Pasquiou, R. Grimm and F. Schreck, Phys. Rev. Lett. 109, 115302 (2012).

[7] G. Reinaudi, C.B. Osborn, M. McDonald, S. Kotochigova and T. Zelevinsky, Phys. Rev. Lett. 109, 115303 (2012).

[8] L.R. Hunter, S.K. Peck, A.S. Greenspon, S.S. Alam and D. DeMille, Phys. Rev. A 85, 012511 (2012).

[9] S.M. Skoff, R.J. Hendricks, C.D.J. Sinclair, J.J. Hudson, D.M. Segal, B.E. Sauer, E.A. Hinds and M.R. Tarbutt, Phys. Rev. A 83, 023418 (2011).

[10] C.H. Wu, J.W. Park, P. Ahmadi, S. Will and M.W. Zwierlein, Phys. Rev. Lett. 109, 085301 (2012).

[11] M. Debatin, T. Takekoshi, R. Rameshan, L. Reichsöllner, F. Ferlaino, R. Grimm, R. Vexiau, N. Bouloufa, O. Dulieu and H.C. Nägerl, Phys. Chem. Chem. Phys. 13, 18926 (2011).

[12] F.M. Spiegelhalder, A. Trenkwalder, D. Naik, G. Kerner, E. Wille, G. Hendl, F. Schreck and R. Grimm, Phys. Rev. A 81, 043637 (2010).

[13] J. Deiglmayr, A. Grochola, M. Repp, K. Mörtlbauer, C. Glück, J. Lange, O. Dulieu, R. Wester and M. Weidemüller, Phys. Rev. Lett. 101, 133004 (2008).

[14] J. Aldegunde, B.A. Rivington, P.S. Żuchowski and J.M. Hutson, Phys. Rev. A 78, 033434 (2008).

[15] J. Aldegunde and J.M. Hutson, Phys. Rev. A 79, 013401 (2009).

[16] B. Friedrich and D. Herschbach, Phys. Chem. Chem. Phys. 2, 419 (2000).

[17] M. Lara, B.L. Lev and J.L. Bohn, Phys. Rev. A 78, 033433 (2008).

[18] B.K. Stuhl, M. Yeo, B.C. Sawyer, M.T. Hummon and J. Ye, Phys. Rev. A 85, 033427 (2012).
[19] A. Micheli, G. K. Brennen and P. Zoller, Nat. Phys. 2, 341 (2006).

[20] G. K. Brennen, A. Micheli and P. Zoller, New J. Phys. 9, 138 (2007).

[21] T. Lahaye, C. Menotti, L. Santos, M. Lewenstein and T. Pfau, Rep. Prog. Phys. 72, 126401 (2009).

[22] J.M. Brown and A. Carrington, Rotational spectroscopy of diatomic molecules (Cambridge University Press, Cambridge, UK, 2003).

[23] R.N. Zare, Angular Momentum: Understanding Spatial Aspects in Chemistry and Physics (John Wiley and Sons, New York, USA, 1988).

[24] C. Yamada, M. Fujitake and E. Hirota, J. Chem. Phys. 91, 137 (1989).

[25] C. Yamada, M. Fujitake and E. Hirota, J. Chem. Phys. 90, 3033 (1989).

[26] J.M. Brown, E.A. Colbourn, J.K.G. Watson and F.D. Wayne, J. Mol. Spect. 74, 294 (1979).

[27] R.N. Zare, A.L. Schmeltekopf, W.J. Harrop and D.L. Albritton, J. Mol. Spect. 46, 37 (1973).

[28] J.M. Brown and A.J. Merer, J. Mol. Spect. 74, 488 (1979).

[29] H. Lefebvre-Brion and R.W. Field, Perturbations in the Spectra of Diatomic Molecules (Academic Press, Orlando, USA, 1986).

[30] S. M. Freund, E. Herbst, R. P. Mariella and W. Klemperer, J. Chem. Phys. 56, 1467 (1976).

[31] M. Yoshimine, J. Chem. Phys. 57, 1108 (1972).

[32] C. R. Schleif and J. B. Delos, Phys. Rev. A 76, 013404 (2007).

[33] I. N. Kozin and R. M. Roberts, J. Chem. Phys. 118, 10523 (2003).

[34] H.P. Büchler, E. Demler, M. Lukin, A. Micheli, N. Prokof'ev, G. Pupillo and P. Zoller, Phys. Rev. Lett. 98, 060404 (2007).

[35] M. Lemeshko, Phys. Rev. A 83, 051402 (2011).

[36] R.M. Wilson, S.T. Rittenhouse and J.L. Bohn, New J. Phys. 14, 043018 (2012). 